$9{ }^{1}$ College of Environmental Science and Engineering, Ministry of Education Key Laboratory of

10 Pollution Processes and Environmental Criteria, Tianjin Key Laboratory of Environmental

11 Remediation and Pollution Control, Nankai University, Tianjin 300350, China

$12{ }^{2}$ College of Environmental Science and Engineering, State Environmental Protection Key

13 Laboratory of Urban Ambient Air Particulate Matter Pollution Prevention and Control, Nankai

14 University, Tianjin 300350, China

15 Number of pages: 19 Number of tables: 1

Number of figures: 10

$21 *$ Corresponding author: (E-mail) zhangtong@nankai.edu.cn. 
Materials

$\mathrm{AgNO}_{3}(>99.5 \%)$ was purchased from Tianjin Chemical Reagent Co. (China), hydroxylammonium chloride $\left(\mathrm{NH}_{2} \mathrm{OH} \cdot \mathrm{HCl}, 99.99 \%\right)$ was purchased from Aladdin Chemistry Co. Ltd. (China), and polyvinylpyrrolidone (PVP, $M \mathrm{w}=58$ 000) was purchased from Macklin

Biochemical Co., Ltd. (China). Ag NMs coated with PVP were synthesized following a

previously established method with modifications. ${ }^{1} \mathrm{ZnO}$ NMs ( $>97 \%$, without coating) were purchased from Sigma-Aldrich (USA). Sodium sulfide nonahydrate $\left(\mathrm{Na}_{2} \mathrm{~S} \cdot 9 \mathrm{H}_{2} \mathrm{O}, 99.99 \%\right)$ was obtained from Aladdin Chemistry Co. Ltd. (China). Human serum albumin (HSA) was purchased from Solarbio Technology Co., Ltd. (China).

\section{Synthesis of Ag NMs Ag}

NMs were synthesized following the method adapted from Lendl and Leopold. ${ }^{1}$ First, 20 $\mathrm{mL}$ of $10 \mathrm{mM} \mathrm{AgNO} 3$ was added into a $180 \mathrm{~mL}$ solution containing $1.67 \mathrm{mM} \mathrm{NH} \mathrm{NH}_{2} \mathrm{OH} \cdot \mathrm{HCl}$ and $3.33 \mathrm{mM} \mathrm{NaOH}$ under vigorous stirring, and the color of the reaction matrix turned yellow immediately. After 2 minutes, $0.3 \mathrm{~g}$ of PVP was added into the system and stirred for 20 hours. Ag NMs were collected by centrifugal ultrafiltration (Amicon Ultra-15 100 kD, Millipore, MA), and rinsed with deionized (DI) water three times. The stock suspension of Ag NMs was stored at $4{ }^{\circ} \mathrm{C}$ in dark prior to use.

\section{Preparation of simulated body fluids}

Simulated saliva contained $0.720 \mathrm{~g} \mathrm{KCl}, 0.220 \mathrm{~g} \mathrm{CaCl}_{2} \cdot 2 \mathrm{H}_{2} \mathrm{O}, 0.600 \mathrm{~g} \mathrm{NaCl}, 0.680 \mathrm{~g}$ $\mathrm{KH}_{2} \mathrm{PO}_{4}, 0.866 \mathrm{~g} \mathrm{Na}_{2} \mathrm{HPO}_{4} \bullet 12 \mathrm{H}_{2} \mathrm{O}, 1.500 \mathrm{~g} \mathrm{KHCO}_{3}, 0.060 \mathrm{~g} \mathrm{KSCN}$ and $0.030 \mathrm{~g}$ citric acid in 1 L DI water adjusted to $\mathrm{pH} 7.1$ with $\mathrm{NaOH}$. Simulated sweat consisted of $5 \mathrm{~g} \mathrm{NaCl}, 1 \mathrm{~mL}$ lactic acid and $1 \mathrm{~g}$ urea in $1 \mathrm{~L}$ DI water adjusted to $\mathrm{pH} 5.1$ with $\mathrm{NaOH}$. Simulated BALF was prepared according to Gamble's solution that have $0.095 \mathrm{~g} \mathrm{MgCl}_{2}, 6.019 \mathrm{~g} \mathrm{NaCl}, 0.298 \mathrm{~g} \mathrm{KCl}, 0.126 \mathrm{~g}$ 
$\mathrm{Na}_{2} \mathrm{HPO}_{4}, 0.063 \mathrm{~g} \mathrm{Na}_{2} \mathrm{SO}_{4}, 0.368 \mathrm{~g} \mathrm{CaCl}_{2} \cdot 2 \mathrm{H}_{2} \mathrm{O}, 0.574$ sodium acetate, $2.604 \mathrm{~g} \mathrm{NaHCO}$ and

$0.097 \mathrm{~g}$ sodium citrate dihydrate in $1 \mathrm{~L}$ DI water adjusted to $\mathrm{pH} 6.6$ with $\mathrm{HCl}$.

\section{Collection and Processing of Biological Fluids}

A well-defined and standardized protocol was used for collection, storage, and processing of all body fluid samples. Saliva, sweat, and BALF were obtained following the protocols as

previously described. ${ }^{2-4}$ Saliva and sweat samples were collected from five healthy adult volunteers at Nankai University, China. The volunteers rinsed their mouths with distilled water and then spit the saliva into $50 \mathrm{~mL}$ centrifuge tubes. For the collection of sweat samples, the volunteers' arms and legs were wiped with 75\% $(v / v)$ alcohol and covered with plastic wraps. The volunteers then ran on treadmill machines for $45 \mathrm{~min}$ and the sweat samples were collected from their skin of arms and legs. BALF samples were obtained according to the approved protocols by Tongji Hospital's review board as well as the informed consent from the patients. Upon collection, the body fluid samples were centrifuged at $2600 \mathrm{~g}$ for $15 \mathrm{~min}$ at $4^{\circ} \mathrm{C}$ to remove debris and cells. The supernatant was then aliquoted and stored at $-80^{\circ} \mathrm{C}$ to avoid protein degradation prior to use. The $\mathrm{pH}$ of saliva, sweat and BALF was 7.1, 5.1 and 6.6, respectively.

\section{Sodium dodecyl sulfate polyacrylamide gel electrophoresis (SDS-PAGE)}

$20 \mu \mathrm{L}$ of protein samples was separated on $4 \%$ to $12 \%$ gradient SDS-PAGE. The molecular weight of the proteins was estimated by comparison with PageRuler ${ }^{\mathrm{TM}}$ Prestained Protein Ladder marker (Fermentas, Burlington, Canada). Gel electrophoresis was performed at $80 \mathrm{~V}$ for 30 minutes and $120 \mathrm{~V}$ for 90 minutes, until the protein bands appeared near the end of the gel. The gels were stained using Coomassie Brilliant Blue staining kit (Sangon Biotech Co., Ltd., Shanghai, China). All gel images were collected using a camera (12 million pixels) and further analyzed using image $\mathrm{J}$ (1.50i version). 


\section{Liquid chromatography-mass spectrometry/mass spectrometry (LC-MS/MS)}

The excised gel bands were left in $1 \mathrm{~mL}$ DI water for $10 \mathrm{~min}$, and washed twice with 25 $\mathrm{mM} \mathrm{NH}_{4} \mathrm{HCO}_{3}$ in $50 \%(v / v)$ acetonitrile $(\mathrm{ACN})$ until the blue color disappeared. The gel bands were then dehydrated with $100 \%(v / v)$ ACN, vacuum dried and immersed in $10 \mathrm{mM}$ DLdithiothreitol (DTT) for $1 \mathrm{~h}$ at $56^{\circ} \mathrm{C}$. Afterward, the DTT solution was removed and $55 \mathrm{mM}$ iodoacetamide was added, followed by incubation at room temperature for $45 \mathrm{~min}$ in the dark. The gel bands were then washed with $25 \mathrm{mM} \mathrm{NH}_{4} \mathrm{HCO}_{3}$ in $50 \%(v / v)$ acetonitrile and dehydrated with $100 \%(v / v)$ ACN. Finally, $20 \mu \mathrm{L}$ trypsin solution $(1 \mu \mathrm{g} / \mu \mathrm{L}$, proteomics grade, Sigma-Aldrich, USA) was added prior to an overnight incubation at $37^{\circ} \mathrm{C}$. The enzymatic hydrolysis was terminated by the addition of $0.1 \%$ formic acid (FA).

After digestion, the peptide mixtures were analyzed by LC-MS/MS using a Q Exactive spectrometry system (Thermo scientific Inc., USA). For peptide separation, the mixtures were applied on a $\mathrm{C} 18$ trap column (Thermo scientific Inc., USA) with DI water $\left(\mathrm{A}: \mathrm{H}_{2} \mathrm{O}, 0.1 \%\right.$ FA) and $\mathrm{ACN}$ (B: ACN, 0.1\% FA) as eluents. An elution program (0-6 min: 5\%-8\% B; 6-40 min: 8\%30\% B; 40-45 min: 30\%-60\% B; $45-48$ min: $60 \%-80 \%$ B; $48-56$ min: 80\% B; 56-58 min: 80\%5\% B; 58-65 min: 5\% B) was executed on an ultimate 3000 nanoLC system (Dionex Inc., USA) with a flow rate of $400 \mathrm{~nL} / \mathrm{min}$. The MS was operated in the positive ion mode and the $\mathrm{m} / \mathrm{z}$ ratio of 350-2000 was scanned. For protein identification, raw data files were converted to Mascot generic format files and searched in National Center for Biotechnology Information (NCBInr) database via Mascot Search (version 2.3.01, Matrix Science, http://www.matrixscience.com/). Mascot searching parameters included trypsin as the proteolytic enzyme with one missed cleavage. The modifications of methionine oxidation were selected. Peptide charge was set to +1 , +2 and +3 . A minimal Mascot score of 50 was set for protein identity validation. 


\section{$91 \quad$ Programming details of random forest classification (RFC)}

93 https://scikit-learn.org/stable/index.html) and consisted of the following steps.

\# Author: Tingting Du 〈dxp0123@mail.nankai.edu.cn〉

\# Step 1: import Library

import numpy as np

import pandas as $\mathrm{pd}$

from sklearn.ensemble import RandomForestClassifier

from sklearn.model_selection import GridSearchCV

from sklearn.model_selection import cross_val_score

from sklearn.metrics import f1_score

\section{\# Step 2: get data}

proteinData $=$ pd.read_excel("C:\\Users $\backslash \backslash \ldots \backslash \backslash S I-X L S X . x l s x "$, sheet_name='Table S5 (or Table S6)', nrows = 100 (or 167), skiprows $=1$ )

$\mathrm{x}=$ proteinData[['Molecular Weight', 'Isoelectric Point', 'GRAVY Value', '\% H-Bonding Relevant Amino Acids', '\% Cysteine Amino Acid']]

$y=\operatorname{proteinData[}$ ['Result $*$ ']

\section{\# Step 3: build a classifier}

$\mathrm{rfc}=$ RandomForestClassifier(n_estimators $=1000$

, oob_score $=$ True

, n_jobs $=-1$

, random_state $=100$

, class_weight $=$ "balanced"

)

\# Step 4: run grid search to select the model hyper-parameters param_grid $=\{$

$$
\text { 'max_depth': np.arange }(1,1+20,1)
$$$$
\text { , 'min_samples_leaf': np.arange(1, } 1+10,1)
$$$$
\text { , 'min_samples_split': np.arange(2, 2+20, 1) }
$$

, 'criterion': ['gini', 'entropy'] 
GS. fit $(x, y)$

print ("Best parameters: In \{\}$"$.format(GS.best_params_))

\# Step 5: run 50 times at random to obtain feature importance and F1 scores of each

132 time

133 importance $=[]$

$134 \mathrm{~F} 1=[]$

135

136

137

138

139

140

141

142

143

144

145

146

147

148

149

150

151

152

153

154

155

156

157

for $i$ in range(50): $\mathrm{rfc}=$ RandomForestClassifier(n_estimators $=1000$

, oob_score $=$ True

, n_jobs $=-1$

, class_weight = "balanced"

, max_depth $=a \# a$ : the value of max_depth selected from

grid search

, min_samples_leaf $=\mathrm{b} \# \mathrm{~b}$ : the value of min_samples_leaf

selected from grid search

, min_samples_split $=\mathrm{c} \# \mathrm{c}$ : the value of

min_samples_split selected from grid search

, criterion = 'gini' or 'entropy'\# use the criterion

selected from grid search

$$
\text { , random_state }=i * 10
$$

)

$$
r f c=r f c . f i t(x, y)
$$

importance.append (rfc.feature_importances_)

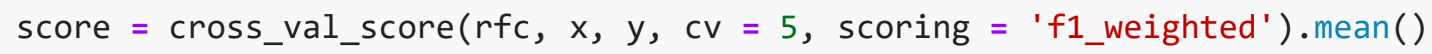

F1. append(score)

importance $=n p \cdot \operatorname{array}(p d$. DataFrame $($ importance $))$

my_matrix $=p d$. concat $([$ pd.DataFrame(importance), pd.DataFrame(F1) $]$, axis=1)

my_matrix.columns $=$ ['Molecular Weight', 'Isoelectric Point', 'GRAVY Value', '\% HBonding Relevant Amino Acids', '\% Cysteine Amino Acid', 'F1 score'] 
np.savetxt('feature importance and F1 for Ag SAg.csv' (or 'feature importance and F1

In addition to RFC, we also tested two other classification methods, k-nearest neighbors

$161(\mathrm{KNN})$ and decision tree classification (DTC). During model validation, the F1-scores of the

$162 \mathrm{KNN}$ and decision tree methods appeared to be lower compared to the F1-scores of the RFC

163 method (Figure S9).

164 Kinetic experiments of HSA adsorption on NMs

165 To assess the adsorption kinetics of HSA on NMs, fixed amount of HSA (50 mg/L) and NMs

166 (1 $\mathrm{mM}$ as metal) were allowed to interact at $150 \mathrm{rpm}$ in a rotary shaker for $12 \mathrm{~h}$ at $\mathrm{pH} 7.4$. The

167 total volume of the interaction solution was maintained as $10 \mathrm{~mL}$. Samples collected at $5 \mathrm{~min}, 2$

$168 \mathrm{~h}, 4 \mathrm{~h}$ and $12 \mathrm{~h}$ were centrifuged and analyzed for the concentrations of the unadsorbed proteins.

169 The adsorbed amount of HSA was calculated based on a mass balance approach. All adsorption

170 experiments were performed in triplicate. And the results showed that $4 \mathrm{~h}$ was enough time to

171 reach adsorption equilibrium (Figure S10). 
Table S7. Summary of adsorption parameters [Freundlich model coefficients $\left(K_{F}\right.$ and $\left.n\right)$ ] of HSA to pristine and sulfidized NMs.

\begin{tabular}{cccc}
\hline $\mathrm{NMs}$ & $K_{\mathrm{F}}\left(\mathrm{mg}^{1-\mathrm{n}} \mathrm{L}^{\mathrm{n}} / \mathrm{mmol}\right.$ NMs as metal $)$ & $n$ & $R^{2}$ \\
\hline $\mathrm{Ag}$ & 0.2479 & 0.9846 & 0.9843 \\
$\mathrm{sAg}$ & 0.3171 & 0.9714 & 0.9968 \\
$\mathrm{ZnO}$ & 0.5892 & 1.1654 & 0.9587 \\
$\mathrm{sZnO}$ & 1.1480 & 1.2930 & 0.9966 \\
\hline
\end{tabular}


(A)

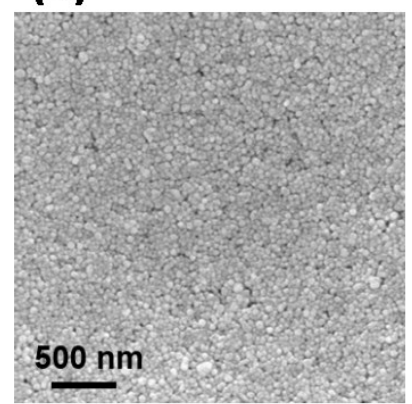

(C)

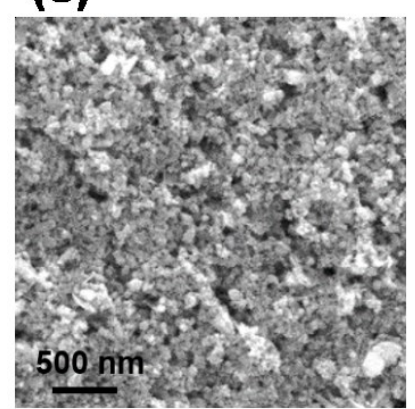

(E)
(B)

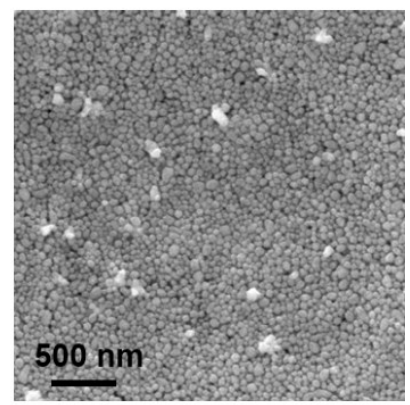

(D)

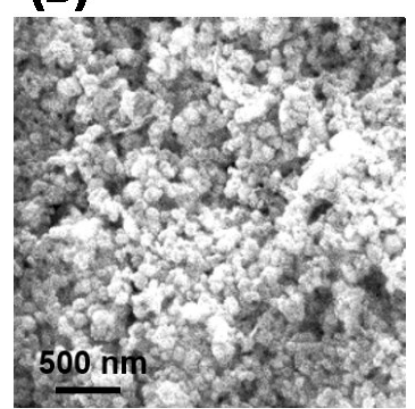

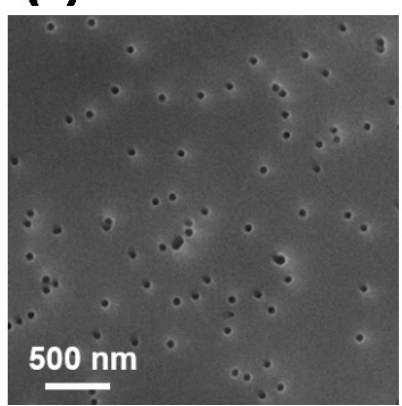

Figure S1. The scanning electron microscopy (SEM) images of the membranes and thin NM films for contact angle measurements: Ag NM (A), sAg NM (B), ZnO NM (C) and sZnO NM (D) samples and pristine membrane (E). 


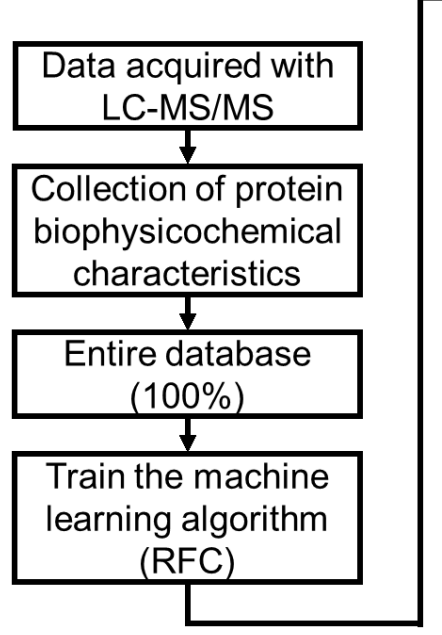

\begin{tabular}{|c|c|c|c|c|}
\hline \multicolumn{5}{|c|}{$\begin{array}{l}\text { 5-fold cross validation } \\
\text { based on grid search }\end{array}$} \\
\hline \begin{tabular}{c|c|c|c|} 
Fold 1 \\
$\mathbf{( 2 0 \% )}$
\end{tabular} & $\begin{array}{l}\text { Fold 2 } \\
\mathbf{( 2 0 \% )}\end{array}$ & $\begin{array}{l}\text { Fold 3 } \\
\mathbf{( 2 0 \% )}\end{array}$ & $\begin{array}{l}\text { Fold 4 } \\
\mathbf{( 2 0 \% )}\end{array}$ & $\begin{array}{l}\text { Fold 5 } \\
\mathbf{( 2 0 \% )}\end{array}$ \\
\hline Train & Train & Train & Train & Test \\
\hline Train & Train & Train & Test & Train \\
\hline Train & Train & Test & Train & Train \\
\hline Train & Test & Train & Train & Train \\
\hline Test & Train & Train & Train & Train \\
\hline & & & & \\
\hline
\end{tabular}

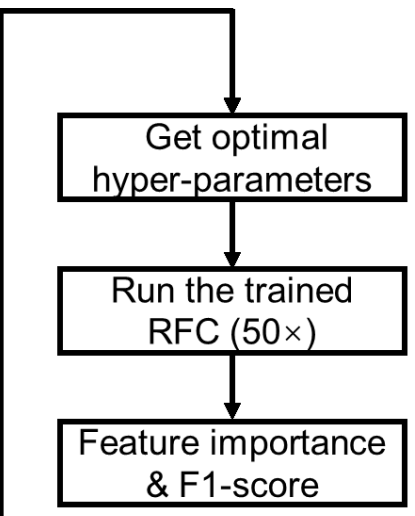

Figure S2. A graphical depiction of data partitioning scheme and random forest classification (RFC) pipeline. This pipeline describes the process step by step to get feature importance from trained RFC. Data acquired by LC-MS/MS was screened and the biophysicochemical characteristics of protein were collected during the pre-processing step. Grid search with 5-fold cross validation was used to automatically select the model hyper-parameters and minimize the generalization error. The trained model was then run and validated 50 times with random dataset partitions and produced performance metrics. 
(A) $\mathrm{Ag}$

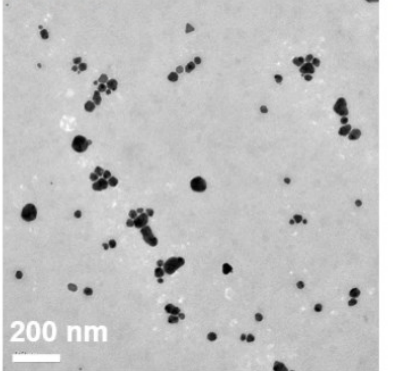

(E) Ag

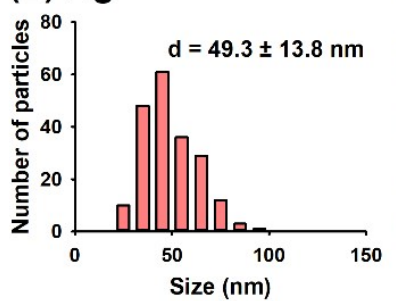

(I) $\mathrm{Ag}$
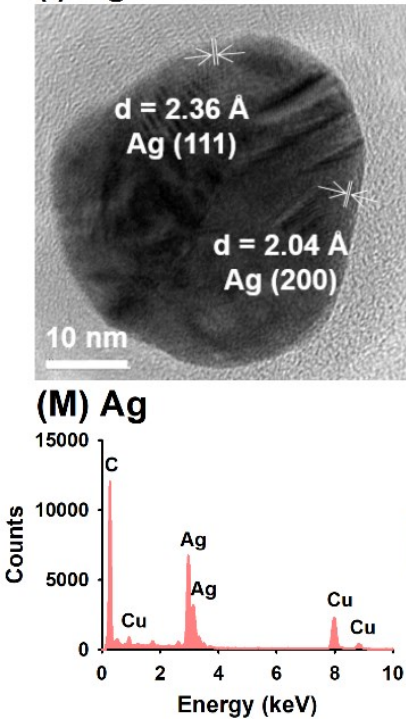

(B) $\mathrm{sAg}$

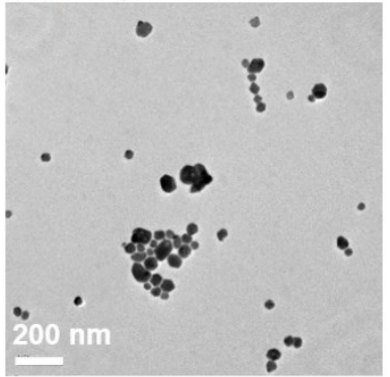

(F) sAg

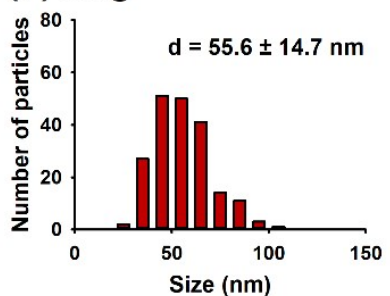

(J) $\mathrm{sAg}$

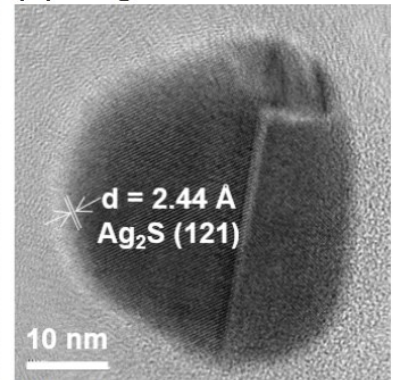

(N) sAg

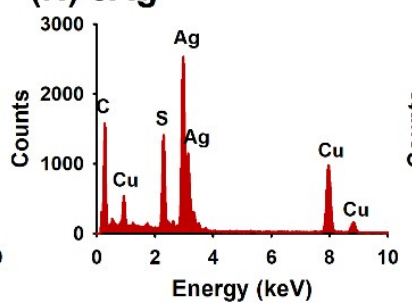

(C) ZnO

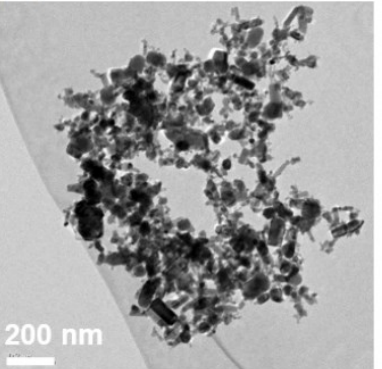

(G) ZnO

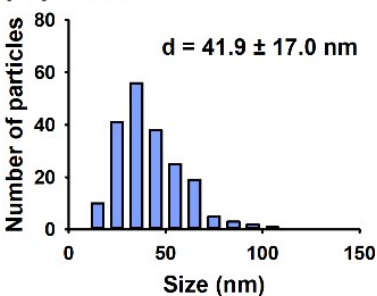

(K) ZnO

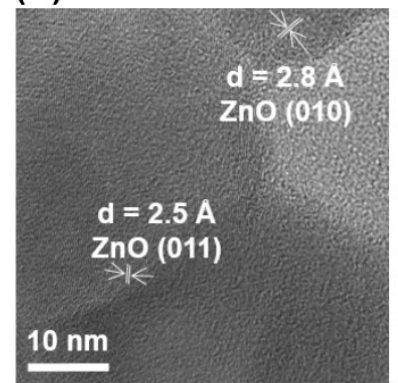

(O) ZnO

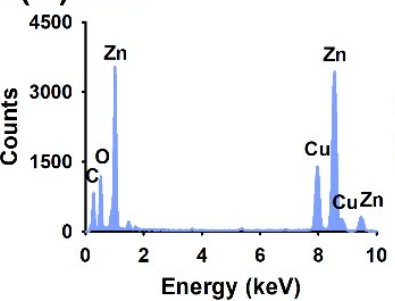

(D) sZno

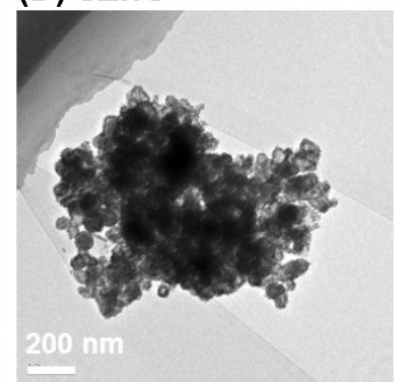

(H) sZno

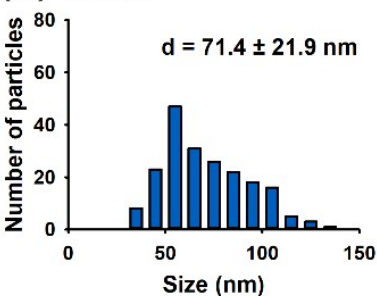

(L) $s Z n O$

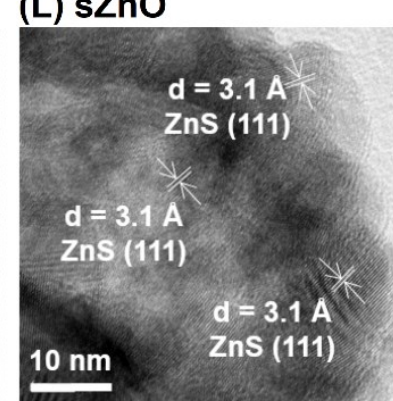

(P) sZno

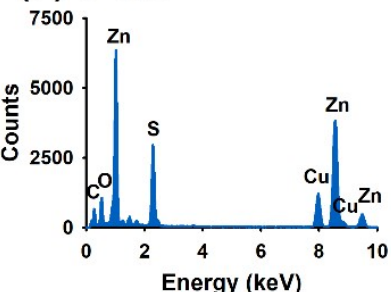

Figure S3. Transmission electron microscope (TEM) images (A-D) and size distribution (E-H) determined from counting 200 particles on 3 separate TEM images of Ag NMs (A, E), sAg NMs (B, F), ZnO NMs (C, G) and sZnO NMs (D, H). High-resolution TEM (HRTEM) images (I-L) and energy dispersive X-ray spectroscopy (EDS) spectra (M-P) of Ag NMs (I, M), sAg NMs (J, $\mathrm{N}), \mathrm{ZnO}$ NMs (K, O), sZnO NMs (L, P). 
(A)

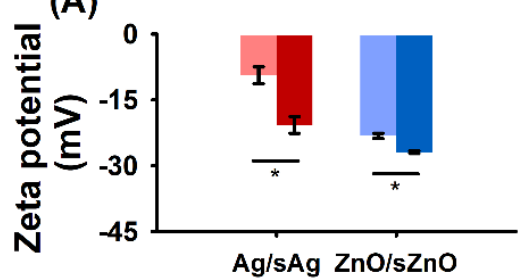

(D)

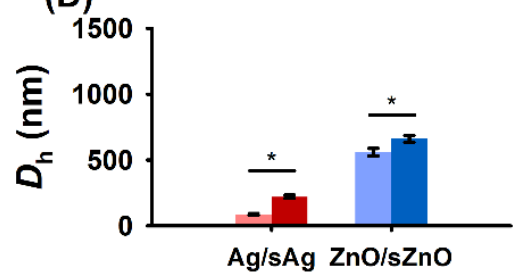

(G)

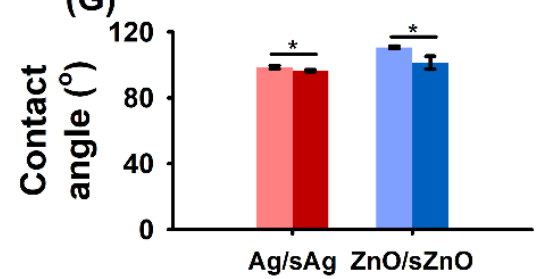

(J)

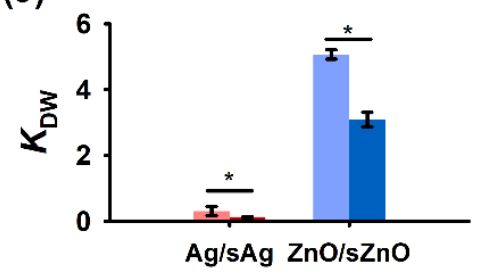

(B)

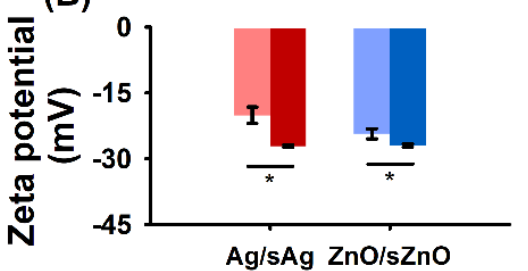

(E)

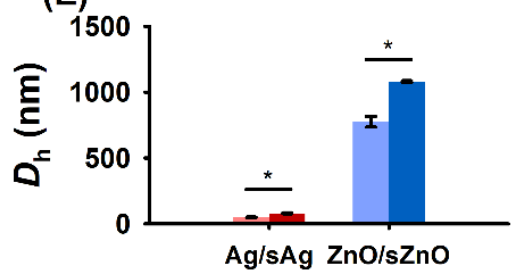

(H)

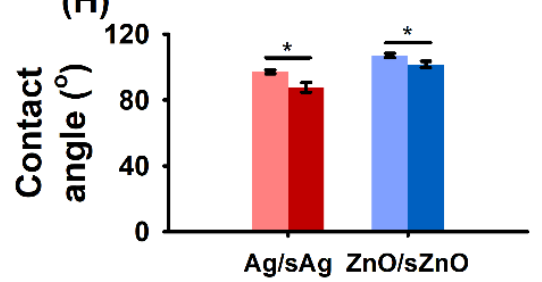

(K)

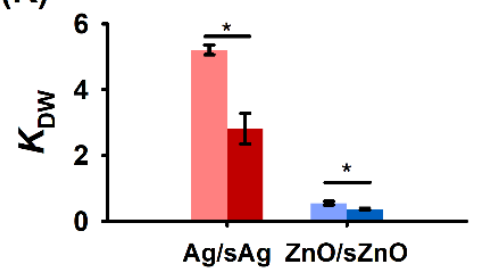

(C)

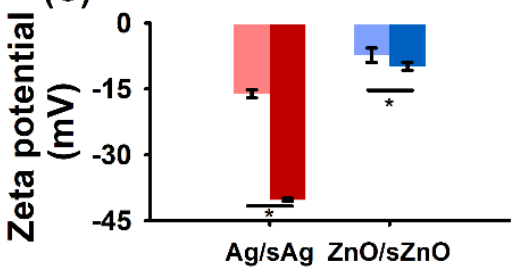

(F)

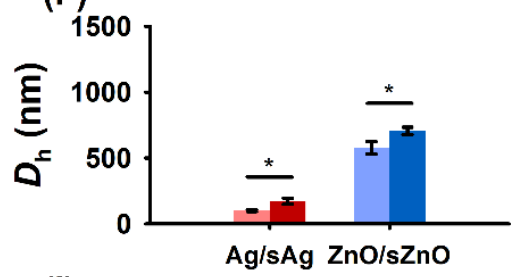

(I)

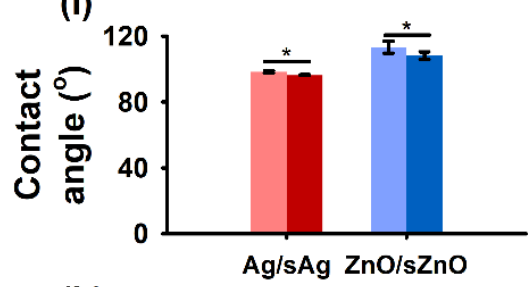

(L)

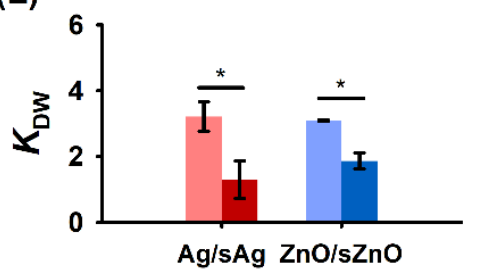

Figure S4. Zeta potential (A-C), hydrodynamic diameter of NMs (D-F), contact angle (G-I) and $K_{\text {Dw }}(\mathrm{J}-\mathrm{L})$ were measured in PBS (A, D), DI water $(\mathrm{G}, \mathrm{J})$, simulated saliva $(\mathrm{B}, \mathrm{E}, \mathrm{H}, \mathrm{K})$ and sweat (C, F, I, L). The error bars represent standard deviations of triplicate samples. Statistical significance between pristine and sulfidized groups: $\left(^{*}\right) p<0.05$. 


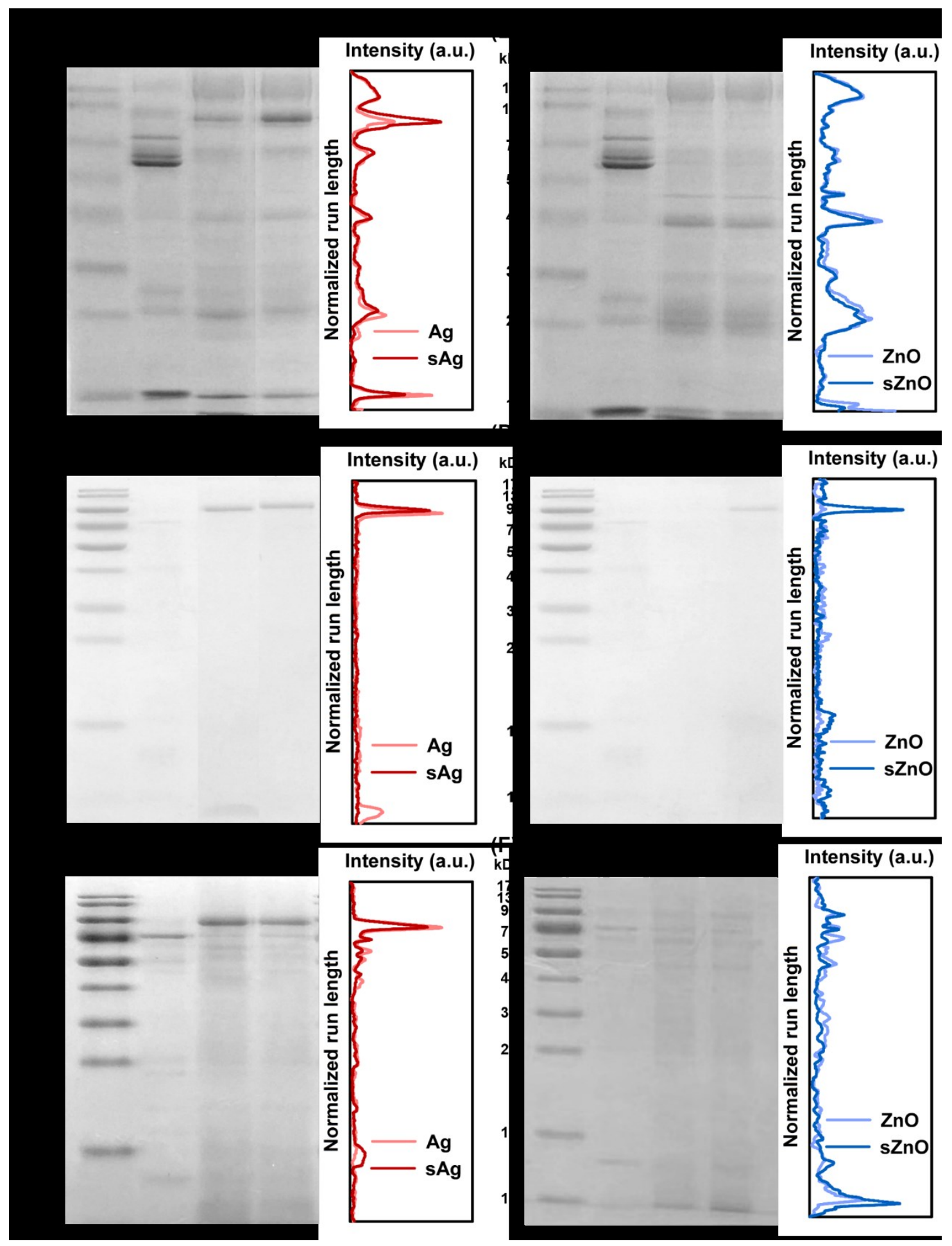

Figure S5. Sulfidation-induced changes in the protein composition of the hard coronas on $\mathrm{Ag}$ NMs (A, C, E) and ZnO NMs (B, D, F) incubated in saliva (A-B), sweat (C-D) and BALF (E-H). Left: SDS-PAGE gels of the markers, body fluids and eluted hard corona samples. Right: intensity of the protein bands across the gel lanes. (Replicate 1) 


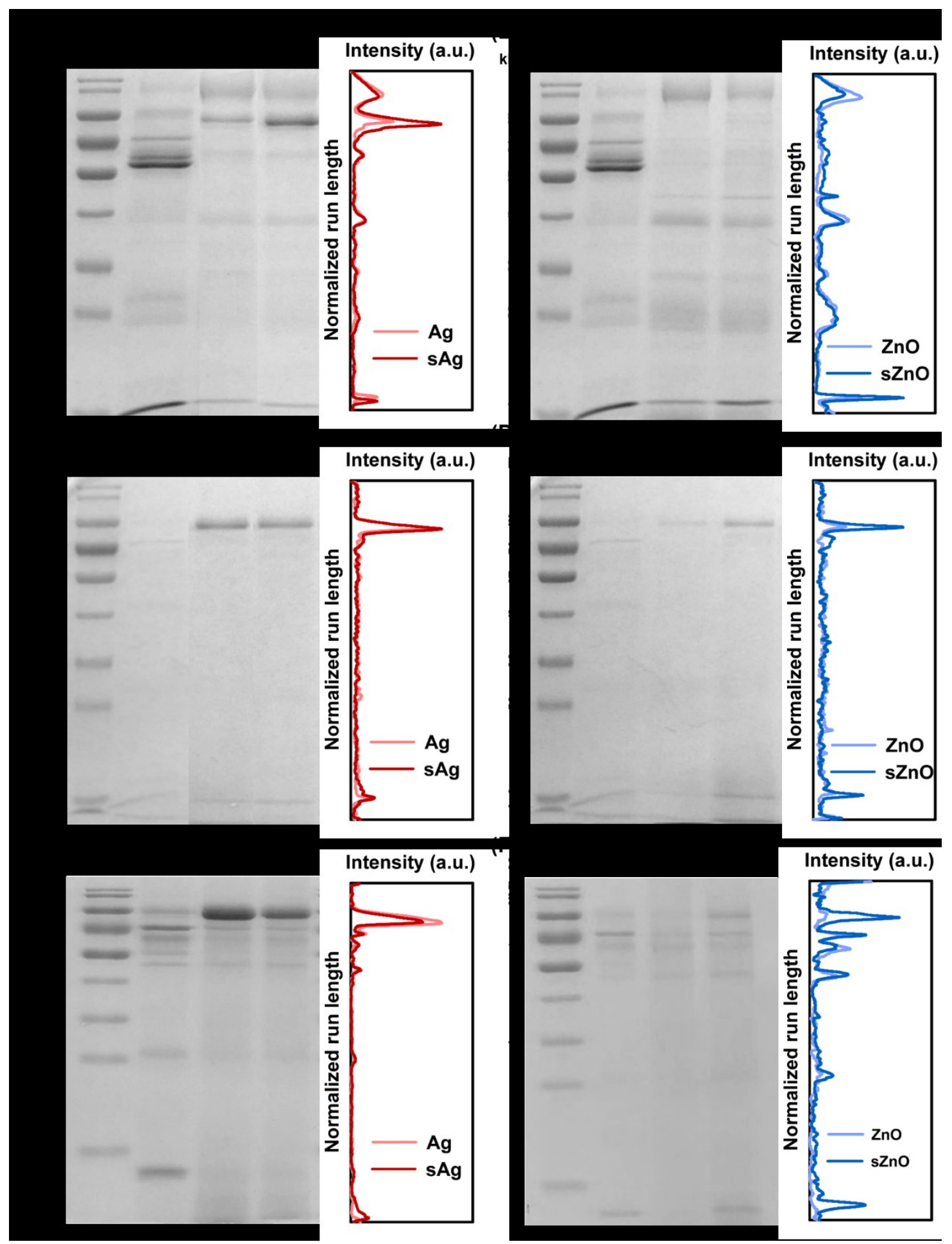

Figure S6. Sulfidation-induced changes in the protein composition of the hard coronas on $\mathrm{Ag}$ NMs (A, C, E) and ZnO NMs (B, D, F) incubated in saliva (A-B), sweat (C-D) and BALF (E-H). Left: SDS-PAGE gels of the markers, body fluids and eluted hard corona samples. Right: intensity of the protein bands across the gel lanes. (Replicate 2) 

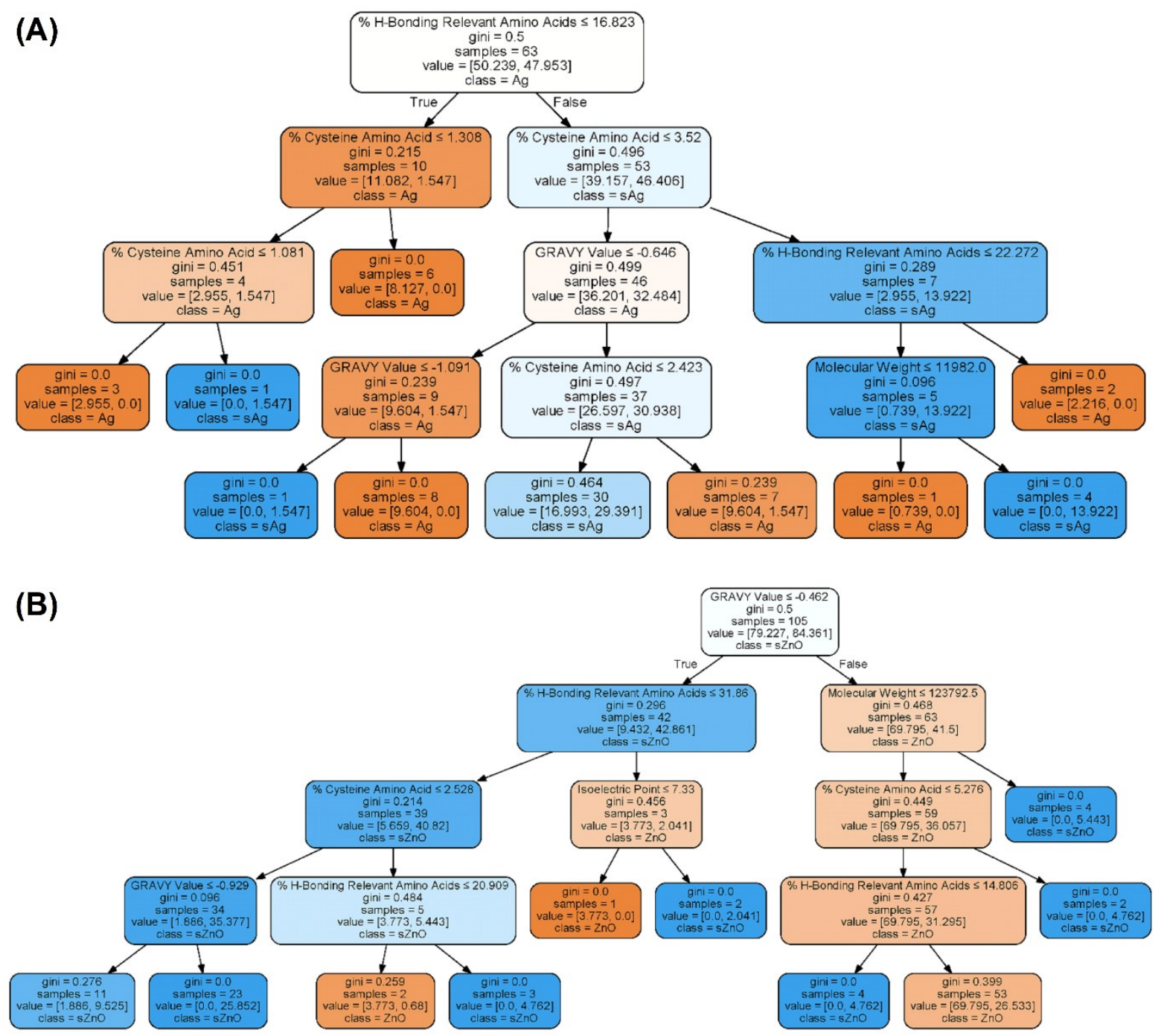

Figure S7. Random decision trees in trained RFC of Ag versus sAg (A) and $\mathrm{ZnO}$ versus sZnO (B). 

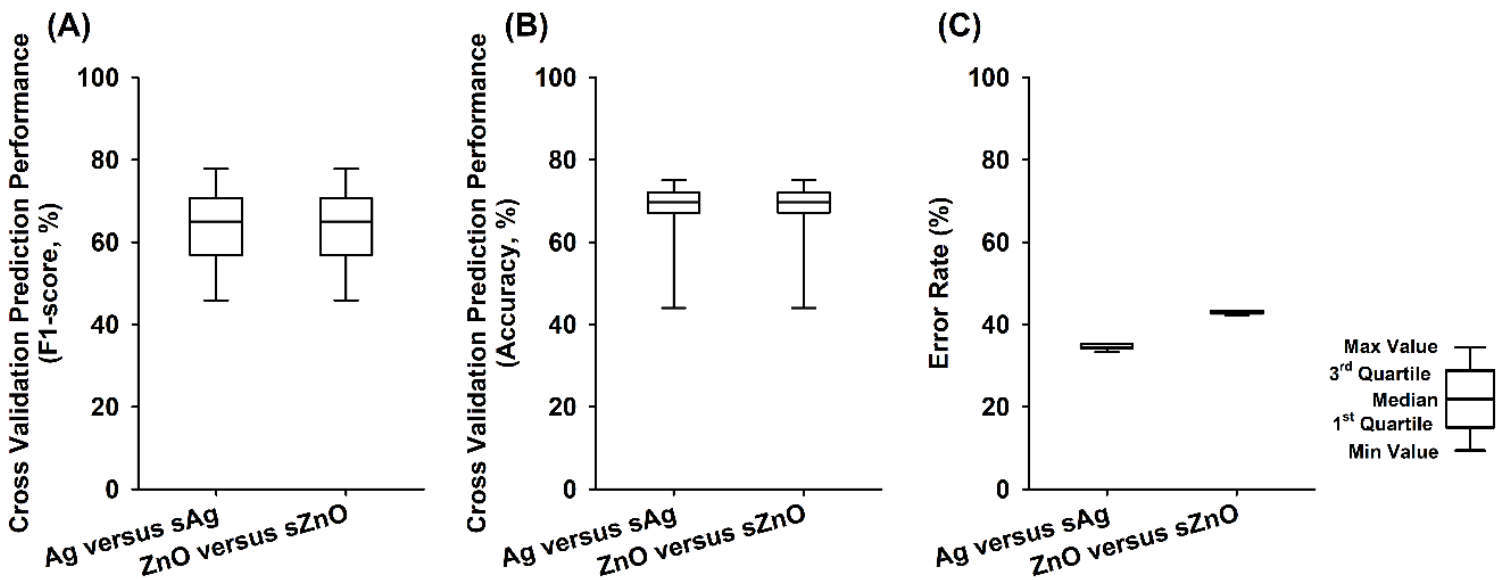

Figure S8. F1-scores (A) and accuracies (B) of 5-fold cross validation and error rates (C) of trained RFC models. 
(A)

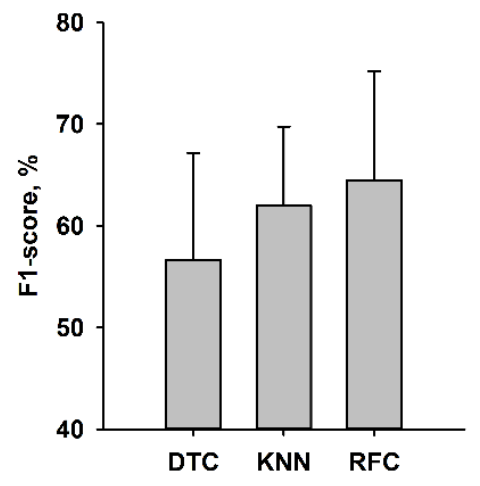

(B)

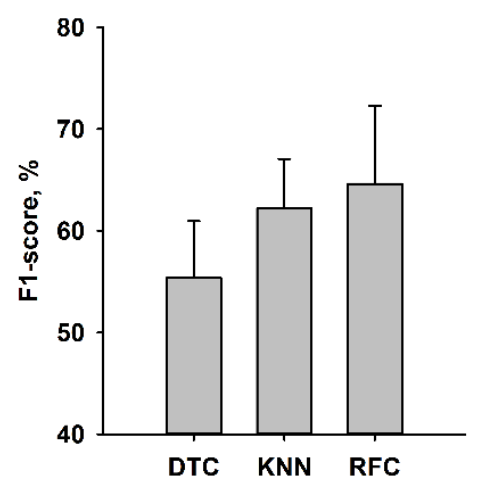

Figure S9. F1-scores of DTC, KNN and RFC models: (A) Ag versus sAg, (B) $\mathrm{ZnO}$ versus sZnO. 


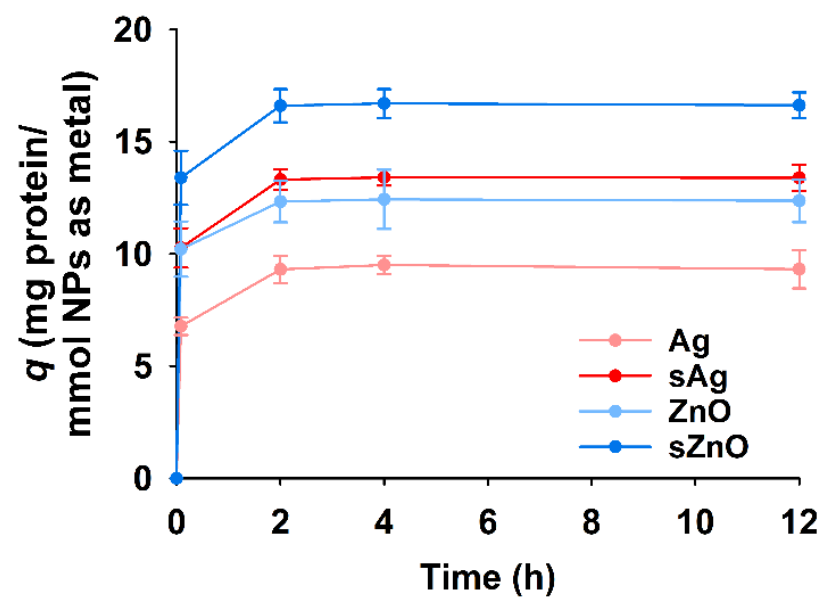

Figure S10. Adsorption kinetics of HSA on pristine and sulfidized Ag and ZnO NMs.

Experimental conditions: $50 \mathrm{mg} / \mathrm{L}$ HSA were exposed to $1 \mathrm{mM}$ (as metal) NMs at $\mathrm{pH} 7.4$ for 5 $\min , 2 \mathrm{~h}, 4 \mathrm{~h}$ and $12 \mathrm{~h}$. The error bars represent standard deviations of triplicate samples. 


\section{REFERENCES}

1. Leopold, N.; Lendl, B., A new method for fast preparation of highly surface-enhanced Raman scattering (SERS) active silver colloids at room temperature by reduction of silver nitrate with hydroxylamine hydrochloride. J. Phys. Chem. B 2003, 107 (24), 5723-5727.

2. Navazesh, M., Methods for collecting saliva. Ann. NY. Acad. Sci. 1993, 694, 72-77.

3. Hooton, K.; Li, L., Nonocclusive sweat collection combined with chemical isotope labeling LC-MS for human sweat metabolomics and mapping the sweat metabolomes at different skin locations. Anal. Chem. 2017, 89 (15), 7847-7851.

4. Kumar, A.; Bicer, E. M.; Morgan, A. B.; Pfeffer, P. E.; Monopoli, M.; Dawson, K. A.; Eriksson, J.; Edwards, K.; Lynham, S.; Arno, M.; Behndig, A. F.; Blomberg, A.; Somers, G.; Hassall, D.; Dailey, L. A.; Forbes, B.; Mudway, I. S., Enrichment of immunoregulatory proteins in the biomolecular corona of nanoparticles within human respiratory tract lining fluid.

Nanomedicine 2016, 12 (4), 1033-1043. 